\title{
GESTÃO E AUTONOMIA NO CURRÍCULO DE EDUCAÇÃO FÍSICA: CAMINHOS PARA A DESCONSTRUÇÃO DA IMAGEM DE "JOGADORES DE BOLA"
}

\author{
GESTIÓN Y AUTONOMÍA EN EL CURRÍCULO DE EDUCACIÓN FÍSICA: \\ CAMINOS PARA LA DESCONSTRUCCIÓN DE LA IMAGEN DE LOS \\ “JUGADORES DE FÚTBOL"
}

\section{MANAGEMENT AND AUTONOMY IN THE CURRICULUM OF PHYSICAL EDUCATION: PATHWAYS FOR THE DECONSTRUCTION OF THE PHYSICAL EDUCATORS AD “FOOTBALL PLAYERS”}

\author{
Allana GLAUCO DA SILVA ${ }^{1}$ \\ Mônica PEREIRA DOS SANTOS ${ }^{2}$ \\ Manoella SENNA ${ }^{3}$
}

RESUMO: Neste artigo, de caráter ensaístico, realizamos um estudo bibliográfico sobre nossas três palavras-chave nas plataformas acadêmicas Scielo e ERIC, bem como um estudo exploratório sobre o currículo de Educação Física da Universidade Federal do Rio de Janeiro, com o objetivo de levantar se as temáticas gestão, autonomia e currículo estão contempladas em sua grade. Partimos da premissa de que a presença destas preocupações na formação inicial de licenciados em Educação Física pode contribuir para minimizar a imagem (negativa) do senso comum sobre educadores físicos como meros "jogadores de bola". Isto porque, conforme a concepção curricular de formação inicial, tais prerrogativas, quando presentes na grade, poderiam ajudar a formar profissionais que não se preocupem apenas com a parte técnica e competitiva da educação física, mas com esta matéria como um campo de saber em si mesmo que pode, por sua vez, contribuir para a formação de profissionais éticos e politizados, mobilizados pela defesa de direitos humanos e do bem comum. Os resultados apontaram a ausência das temáticas na grade curricular do curso, assim como uma baixa quantidade na pesquisa bibliográfica o que, para nós, pode ser um dos indicativos da imagem negativa destes profissionais no senso comum.

PALAVRAS-CHAVE: Educação física. Gestão. Autonomia. Currículo.

RESUMEN: En este artículo ensayístico, llevamos a cabo un estudio bibliográfico sobre las publicaciones qué contienen nuestras palabras clave en las plataformas

${ }^{1}$ Universidade Federal do Rio de Janeiro (UFRJ), Rio de Janeiro - RJ - Brasil. Graduanda em Educação Física. ORCID: https://orcid.org/0000-0001-8863-0688. E-mail: glaucoallana@gmail.com

${ }^{2}$ Universidade Federal do Rio de Janeiro (UFRJ), Rio de Janeiro - RJ - Brasil. Professora Associada. Departamento de Fundamentos da Educação. ORCID: https://orcid.org/0000-0002-7057-7804. E-mail: monicapes@gmail.com

${ }^{3}$ Universidade Federal do Rio de Janeiro (UFRJ), Rio de Janeiro - RJ - Brasil. Doutoranda em Educação. Orientadora Educacional do Colégio Garriga de Menezes. ORCID: https://orcid.org/0000-0002-46560359. E-mail: manoellasenna@hotmail.com 
Scielo y Eric, así como un estudio exploratorio sobre el currículo de educación física de la Universidad Federal de Rio de Janeiro. El objetivo fue saber si los temas de la gestión y de la autonomía están incluidos en su programa. Partimos de la premisa de que la presencia de estas preocupaciones en la formación inicial de licenciados en educación física puede contribuir a minimizar la imagen (negativo) del sentido común acerca de profesores de educación física como simple "jugadores de fútbol". Esto es porque, como el diseño curricular de la formación inicial, tales prerrogativas, cuando están presentes en el currículo, podrían ayudar a formar a profesionales que no se preocupen solo con la parte técnica y competitiva de la educación física, pero también con este asunto como un campo de saber en sí mismo y que él, por su vez, contribuya a la formación de profesionales éticos y políticos, movilizados para la defensa de los derechos humanos y el bien común. Los resultados mostraron la ausencia de los temas en el currículo del curso, e poca producción académica que junte los asuntos, y esto, para nosotros, puede ser una de las indicaciones del porqué de la imagen negativa de estos profesionales en el sentido común.

PALABRAS CLAVE: Educación física. Gestión. Autonomía. Currículo.

ABSTRACT: In this essay, we performed a bibliographical study on literature that presented our key words together in the academic platforms of Scielo and Eric, as well as an exploratory study on the curriculum of physical education licentiateship course of the Federal University of Rio de Janeiro. The aim was to see if the themes of management and autonomy are included in its grid. We set off from the premise that the presence of these concerns in the initial training of graduates in physical education licentiateship course can contribute to minimize the (negative) image of common sense about physical education teachers as mere "football players". This is so because, depending on the curricular design of initial formation, such prerogatives, when present in the grid, could help train professionals not to worry just with the technical and competitive part of the physical education. It could also train them to see teaching physical education as a field of knowledge in itself that can contribute to the formation of ethical and political professionals, mobilized by the defense of human rights and the common good. The results showed the absence of such themes in the curriculum of the course and a low incidence of articles that bring the key words together, which for us can be an indicator of the reasons why the negative image of these professionals exist in the common sense.

KEYWORDS: Physical Education. Management. Autonomy. Curriculum.

\section{Introdução}

O currículo, e mais especificamente, a grade curricular, é um norte na formação de todos os alunos, por mais que, em alguns casos, ela esteja extremamente desatualizada e não corresponda à realidade vivida pelos alunos. Por vezes, nos deparamos com a falta de preparo de alunos formados com algumas questões muito importantes e que passam sem a devida atenção em todos os âmbitos educacionais 
quando são testados na prática. Embora a ideia de "qualidade" não esteja ligada apenas ao currículo, mas também a diversos fatores que, em conjunto, fazem ou não a diferença na formação dos alunos, compreendemos que a questão curricular afeta o ensino e o desenvolvimento do saber no período em que o estudante está sendo apresentado a temas até então nunca vistos.

Desde os primórdios da história, aprendemos que a civilização sempre encontrou meios de sobreviver. Para resolver o problema com o inverno e baixa temperatura, o ser humano conseguiu fazer fogo; posteriormente, a necessidade de transitar por distâncias longas o fez domesticar animais, a fim de evoluir, sobreviver e construir uma civilização melhor a cada dia (HARARI, 2017). Quando paramos para pensar na época da pré-história podemos observar o quanto o ser humano era livre para descobrir, tentar, errar, e, finalmente, acertar. Os erros e os acertos daquela época possibilitaram o avanço da humanidade.

Um dos problemas do currículo de licenciandos em educação física dentro das universidades, escolas e afins, é que na maioria das vezes ele não prevê o desenvolvimento da autonomia e da gestão por parte do aluno, como um dos principais focos da formação. Os alunos estão cada vez mais padronizados e estáticos dentro da sala de aula. Para piorar, a satisfação dos alunos nas aulas não é o foco principal do professor, fazendo com que na maioria das vezes a escola/universidade seja um lugar desmotivante e insatisfatório.

Segundo Ryan e Deci (2000, p. 68):

Especificamente, fatores que melhoram contra os que pioram a motivação intrínseca, a auto-regulação e o bem estar têm sido examinados. Os achados têm levado à postulação de três necessidades psicológicas inatas - competência, autonomia e relacionamento - as quais, quando satisfeitas, melhoram a auto motivação e a saúde mental e, quando prejudicadas, levam à diminuição da motivação e do bem estar (tradução livre). ${ }^{4}$

Neste sentido, o presente artigo apresenta um estudo exploratório do currículo de Educação Física da Universidade Federal do Rio de Janeiro, com o objetivo de levantar se tais temáticas estão contempladas em sua grade curricular e discutir em que medida sua presença pode interferir positivamente na desconstrução da imagem

${ }^{4}$ Do original, em inglês: Specifically, factors have been examined that enhance versus undermine intrinsic motivation, self-regulation, and well-being. The findings have led to the postulate of three innate psychological needs--competence, autonomy, and relatedness-which when satisfied yield enhanced selfmotivation and mental health and when thwarted lead to diminished motivation and well-being. 
negativa, ou em que medida sua ausência pode interferir negativamente para alimentar a imagem do senso comum de educadores físicos como meros "jogadores de bola".

Iniciaremos nossa escrita com uma breve descrição do que consideramos por gestão, autonomia e currículo. E, seguida, descreveremos o tipo de metodologia empregado no estudo bibliográfico e como realizamos o levantamento das ementas no currículo do curso de licenciatura em Educação Física da UFRJ. Por fim, discutiremos sua ausência ou presença, bem como defenderemos nosso argumento de que uma eventual ausência seja prejudicial à imagem do profissional que se forma neste curso, e porquê.

\section{Conceitos centrais: Gestão, Autonomia e Currículo}

São variados os autores que trabalham estas três temáticas. Para nossos propósitos, nos ateremos àqueles que melhor "conversam" com nossa perspectiva de educação e formação de profissionais da educação, ou seja, uma perspectiva mais alinhada a um pensamento emancipatório. Por pensamento emancipatório na formação de educadores, queremos dizer aquelas linhas de formação que pensam os papéis dos educadores como mediadores de importantes processos de uma formação humana cidadã, vinculada a Direitos Humanos e libertária: psicológicos (cognitivos, afetivos, linguísticos, físicos), políticos, sociais e culturais. Assim, traremos para nossa discussão autores como Paro, Freire, Silva e outros.

\section{Da Gestão}

Nesta seção é importante ressaltar o que entendemos por gestão para, em seguida, relacioná-la ao campo da educação e qual sua importância para se pensar a formação de professores. Gestão, para Dias (2002) constitui um termo que apresenta diversas concepções e, sendo assim, é fundamental delimitar o campo pelo qual se lê e entende a gestão. Vale ressaltar que, segundo o autor, gestão e administração são conceitos que advindos dos processos de organização da produção e do trabalho, que perpassam o cenário educacional, principalmente devido às demandas e transformações da sociedade. Esses conceitos de gestão e de administração, têm sido muitas vezes utilizados como se eles significassem a mesma coisa e/ou processo, não se atentando às suas especificidades. 
Desse modo, entendemos como Dias (2002) que a administração é incorporada pela gestão, sendo uma das funções necessárias para seu desempenho. No que tange ao campo educacional, compreendemos, a partir de Paro (1998, 2012), a gestão como uma mediação dos processos educacionais, que necessita incorporar conceitos da administração, porém não deixando de lado as especificidades do trabalho pedagógico. Vale ressaltar, como alerta Paro (2015), que não se trata de reforçar a dicotomia pedagógico versus administrativo, mas entender que ambos preconizam a mediação para uma finalidade, sendo ela a educação do aluno.

Sendo a gestão a mediação de processos educacionais, quando pensamos a escola e atuação dos sujeitos nela envolvidos, defendemos que o lugar da gestão não corresponde apenas à direção da escola, mas relaciona-se a outros sujeitos que participam desse processo como, por exemplo, o coordenador pedagógico, o orientador educacional, o secretário escolar - que compõem a chamada equipe de gestão escolar e, ainda, o professor, que organiza e medeia processos dentro de sala de aula. Acreditamos, de acordo com Senna (2017), que a gestão é potencialmente mediadora de políticas, práticas e culturas tanto de inclusão quanto de exclusões. Sendo assim, uma gestão na perspectiva da inclusão em educação, por exemplo, quando pautada no bemestar de todos e no objetivo de uma educação de qualidade, pode construir culturas, desenvolver políticas e orquestrar práticas que lutem a favor da aprendizagem e da participação dos atores desse processo: alunos, funcionários, professores e gestores.

Dito isto, quando discutimos a formação de professores, é necessário compreender a importância de uma formação e de um currículo, portanto, que contemplem questões relacionadas à gestão. Isso porque, segundo cremos, é fundamental ao professor compreender e potencializar seu papel enquanto gestor e mediador de culturas, políticas e práticas mais autônomas, emancipatórias, inclusivas.

\section{Da Autonomia}

"Auto" vem de auto em grego, que significa "de si mesmo", e de nomos, que significa lei. Juntos, teríamos "aquele que estabelece suas próprias leis". Assim, autonomia seria a capacidade de um indivíduo racional tomar uma decisão não forçada baseada nas informações disponíveis. Paulo Freire (1996), um dos referenciais do tema autonomia dentro da educação, critica as formas de ensino tradicionais. $\mathrm{O}$ autor defende uma pedagogia fundada na ética, no respeito, na dignidade e na autonomia do educando, 
além de mostrar o quão importante é uma escola que instigue o pensamento e que saiba acima de tudo que é necessário existir um processo de aprendizado mútuo.

Partindo deste pressuposto, a educação, em todos os níveis e modalidades, deveria ser um âmbito inclusivo e que priorizasse a todos de maneira a instigar o pensamento autônomo. Para Freire, em sua proposta educacional que visa a autonomia do aluno, a procura pela transformação do educando em sujeito é o que implica na promoção da autonomia. Ele propõe, entre outras coisas, uma alfabetização e uma educação que levem à tomada de consciência da própria condição social do indivíduo. A conscientização possibilita a transformação social, fazendo com o que o aluno não se submeta cegamente às normas que a sociedade impõe e sim, por uma decisão fundamentada em seu pensamento crítico e ético.

É importante pensar, neste sentido, que inclusão, termo esse que não é apenas atrelado a pessoas com deficiência e sim a toda e qualquer pessoa em situação ou em risco de exclusão, representa uma luta por condições igualitárias de existência para todos que vivem na sociedade (SANTOS, 2013). É importante que todos entendam que a diferença não pode ser usada como justificativa para desqualificar, minimizar o valor ou excluir alguém, muito pelo contrário. A sociedade é múltipla e soma valências justamente porque ninguém é igual a ninguém. Por isso mesmo, deveríamos aprender, ao longo dos anos, a considerar o que há de melhor em cada um para nos desenvolvermos e evoluirmos melhor como parte de um todo. No entanto, indagamos: em que medida isto pode ser feito quando temos, no Brasil, currículos que vão na contramão desta ideia e que se prendem mais à quantidade do que à qualidade de conteúdos?

\section{Do Currículo}

Segundo Saviani (2016, p. 55):

Currículo é entendido comumente como a relação das disciplinas que compõem um curso ou a relação dos assuntos que constituem uma disciplina, no que ele coincide com o termo programa. Entretanto, no âmbito dos especialistas nessa matéria tem prevalecido a tendência a se considerar o currículo como sendo o conjunto das atividades (incluído o material físico e humano a elas destinado) que se cumprem com vistas a determinado fim. Este pode ser considerado o conceito ampliado de currículo, pois, no que toca à escola, abrange todos os elementos a ela relacionados. 
Deste modo, para nós, o currículo não deve massacrar o aluno a fim de objetivos quantitativos e de resultados pré estabelecidos de notas e avaliação. Pensando assim, a importância de gerir o processo de ensino e aprendizagem dentro da sala de aula, fomentando a autonomia do aluno e o instigando para além de um conteúdo definido, é a chave para estabelecer um elo de confiança e interesse na relação aluno-professor uma vez que, desta forma, o aluno entende que o professor está ali não apenas para "passar a matéria" com cópia, ditado e "rolando a bola", mas, como uma pessoa que se importa com ele.

Nesta concepção de currículo, no momento em que o indivíduo é incluído na sala de aula, por exemplo, ele ganha a liberdade de escolha. O educador, assim, faz com que aquele aluno possa decidir sobre o que diz respeito a ele, da melhor maneira para ele, de acordo com o que ele acha. A partir do momento em que o professor cria um vínculo que estabeleça liberdade de escolha com esse aluno, ambos, aluno e professor, conseguem escolher o conteúdo que seria mais interessante e, consequentemente, mais prazeroso e melhor aproveitado. Em resumo, o educador deve possibilitar que o aluno entenda e compreenda o que é o melhor para ele, oferecendo direito de escolha. Por outro lado, quando observamos recém-licenciados em Educação Física em nossa universidade (UFRJ), nem sempre é isso que constatamos. Temos visto muitos formados reproduzindo uma visão tecnicista de educação em suas práticas pedagógicas, o que muito nos preocupa. Assim, perguntamo-nos se esta hipótese se confirma quando analisamos produções científicas que conjuguem currículo, educação física, autonomia e gestão e quando buscamos estas conjugações na grade curricular da licenciatura em Educação Física da UFRJ: as produções costumam discutir estas temáticas juntas? O currículo da UFRJ as trabalha conjugadamente? Caso negativo, o que isto pode significar em relação ao desenvolvimento de profissionais autônomos, gestores de excelências de suas próprias práticas, defensores de saberes e fazeres compartilhados e de uma formação humana emancipatória e que reflita sobre o combate às desigualdades e sobre a importância em se aproximar, desde a formação inicial, os professores com os Direitos Humanos?

\section{Metodologia}

Como foi dito anteriormente, este artigo se constitui em um ensaio e se caracteriza por um estudo exploratório, tendo em vista as poucas produções encontradas 
que congregassem todos os nossos temas juntos. Por este motivo, e também porque defendemos o argumento (ainda hipotético), a saber, o de que a inserção de conteúdos sobre gestão e autonomia no currículo do profissional licenciado em educação física pode ajudá-lo a reverter uma visão negativa do senso comum a respeito de seu perfil profissional, este capítulo se caracteriza como de cunho ensaístico.

Para Révillion (2003, p. 24), o estudo exploratório pode ser usado para os seguintes objetivos:

- familiarizar e elevar a compreensão de um problema de pesquisa em perspectiva;

- ajudar no desenvolvimento ou criação de hipóteses explicativas de fatos a serem verificados numa pesquisa causal.

- auxiliar na determinação de variáveis a serem consideradas num problema de pesquisa;

- verificar se pesquisas semelhantes já foram realizadas, quais os métodos utilizados e quais os resultados obtidos, determinar tendências, identificar relações potenciais entre variáveis e estabelecer rumos para investigações posteriores mais rigorosas;

- investigar problemas do comportamento humano, identificar conceitos ou variáveis e sugerir hipóteses verificáveis.

Neste capítulo, recorremos a este design metodológico particularmente porque queremos nos familiarizar com o assunto que, segundo verificamos, ainda está pouco conhecido e, com isso, alimentar (ou não) nossa hipótese a respeito da presença ou ausência de aspectos relativos aos temas da gestão e da autonomia no currículo da formação de educadores físicos e seus impactos na imagem e nas práticas do profissional de educação física. Assim, fizemos um levantamento bibliográfico em duas grandes bases de artigos e produções científicas (como será visto na próxima seção), tendo em vista sabermos, de início, se os assuntos aqui enfocados, em conjunto, têm aparecido em tais produções. Com base nestes achados, realizamos nossa análise.

De modo complementar, uma escrita ensaística é aquela em que, segundo Meneghetti (2011, p. 321): “[...] a orientação é dada não pela busca das respostas e afirmações verdadeiras, mas pelas perguntas que orientam os sujeitos para as reflexões mais profundas". Desta forma, aqui nos orientamos pela indagação que nos move a respeito do tema: gestão e autonomia, se inseridos no currículo de formação inicial, poderiam contribuir para se desfazer uma visão negativa do senso comum a respeito de educadores físicos? O caráter ensaístico de nossa escrita a respeito desta questão significa, na contramão de uma visão mais "tradicional" (cartesiana) de ciência, admitir que provavelmente não fecharemos o capítulo com uma conclusão efetiva sobre o 
assunto, mas pelo menos poderemos lançar questões que inspirem a novos estudos sobre a temática.

\section{Resultados e discussão}

Para entendermos melhor se (e, se sim, de que forma) o currículo do curso de Licenciatura em Educação Física da UFRJ contém aspectos vinculados aos temas gestão e autonomia, fizemos um levantamento e analisamos as ementas das 38 disciplinas do currículo atual do referido Curso, além de termos feito uma vasta busca de artigos no Scientific Education Library Online $\left(\right.$ SCIELO $\left.^{5}\right)$ e no Education Resources Information Center $\left(\mathrm{ERIC}^{6}\right.$ ), dois importantes bancos de dados, com as nossas palavras chave e buscando publicações dos últimos 5 anos (2015-2019). A pesquisa realizada na Plataforma Scielo encontrou os seguintes dados:

\begin{tabular}{|c|c|}
\hline Palavras chaves da busca no SCIELO & Resultados \\
\hline Educação Física + Gestão + Autonomia + Currículo & $\mathbf{0}$ \\
\hline Educação Física + Gestão + Autonomia & $\mathbf{0}$ \\
\hline Educação Física + Gestão & 4 \\
\hline Após Corte por datas & 3 \\
\hline Após leitura dos Resumos & 2 \\
\hline
\end{tabular}

\begin{tabular}{|c|c|}
\hline Educação Física + Autonomia & $\mathbf{5}$ \\
\hline Após corte por datas & 3 \\
\hline Após leitura dos Resumos & 1 \\
\hline
\end{tabular}

\begin{tabular}{|c|c|}
\hline Educação Física + Currículo & $\mathbf{1 1 7}$ \\
\hline Após corte por data & 44 \\
\hline Após leitura dos Resumos & 4 \\
\hline
\end{tabular}

${ }^{5}$ Scientific Electronic Library Online (Biblioteca Científica Eletrônica Online)

${ }^{6}$ Education Resources Information Center (Centro de Informação sobre Recursos Educacionais) 
TOTAL GERAL FINAL $(0+0+2+1+4)$

Consideramos apenas artigos de 2015 até a presente data e após a leitura dos resumos obtivemos um total de 2 artigos que permeiam os assuntos educação física + gestão e apenas 1 artigo sobre educação física + autonomia.

Na plataforma ERIC, obtivemos os seguintes resultados:

\begin{tabular}{|c|c|}
\hline Palavras chaves da busca no ERIC (SEM FILTROS) & Resultados \\
\hline Physical Education + Education Management + Autonomy + Curriculum & 12 \\
\hline Physical Education + Education Management + Autonomy & 25 \\
\hline Physical Education + Education Management & 51 \\
\hline Physical Education + Autonomy & 210 \\
\hline Physical Education + Curriculum & 3623 \\
\hline
\end{tabular}

\begin{tabular}{|c|c|}
\hline Palavras chaves da busca no ERIC (COM FILTRO POR DATA) & Resultados \\
\hline Physical Education + Education Management + Autonomy + Curriculum & 4 \\
\hline Physical Education + Education Management + Autonomy & 12 \\
\hline Physical Education + Education Management & 11 \\
\hline Physical Education + Autonomy & 86 \\
\hline Physical Education + Curriculum & 552 \\
\hline Palavras chaves da busca no ERIC (APÓS LEITURA DO RESUMO) & Resultados \\
\hline Physical Education + Education Management + Autonomy + Curriculum & 0 \\
\hline Physical Education + Education Management + Autonomy & 0 \\
\hline Physical Education + Education Management & 3 \\
\hline Physical Education + Autonomy & 22 \\
\hline Physical Education + Curriculum & 59 \\
\hline TOTAL GERAL FINAL (0+0+3+22+59) & $\mathbf{8 4}$ \\
\hline
\end{tabular}

A partir dos resultados apresentados é possível analisar que a maioria dos artigos faz crítica ao sistema educacional em diferentes instâncias que vão desde a educação infantil até a formação acadêmica. Importante ressaltar a "globalização" da crítica, pois, 
é possível encontrar a questão em diversos países como: Canadá (KILBORN; LORUSSO; FRANCIS, 2015), Colômbia e Chile (LARA; STAREPRAVO; MIRANDA; SOUZA, 2015), Nova Zelândia (KIRSTEN, 2016), Turquia (BOZYIGIT, 2017), Dinamarca (SVENDSEN, 2016), Escócia (MITCHELL; GRAY; INCHLEY, 2015), Brasil (BETTIA; KNIJNIKB; VENÂNCIO; NETO, 2015), dentre outros. A formação acadêmica, por sua vez, é criticada em diferentes áreas, como por exemplo na da educação e na de saúde, expondo pontos antigos de uma Educação Física Militarista que tinha como objetivo apenas o preparo e desenvolvimento do corpo humano (NOGUEIRA; BOSI, 2016). No campo da educação por sua vez, pode-se observar a defasagem curricular como principal viés na formação. De acordo com Neves (1999, p. 108), “a escola vive uma situação problemática ao nível da sua gestão pedagógica e administrativa" e isso se dá por diversos fatores, inclusive pela desorganização do currículo dentro das instituições de ensino.

Outro dado interessante se deu partir da quantidade de menções a “autodeterminação". Segundo Ryan e Deci (2000) A teoria da autodeterminação é trazida como forte aliada para um currículo que propõe a autonomia, assim como a singularidade e o cuidado com o sentimento do indivíduo como braços fundamentais para a educação. Trazendo um pouco mais desta teoria podemos perceber que ela se está vinculada a 4 pontos, são eles: Fatores sociais, Mediadores, Motivação e Consequência. Esses tópicos, por sua vez, estão ligados a outros pontos, como: relações, competência, apoio à autonomia, satisfação, relações, motivações intrínsecas, extrínsecas, desmotivações, afeto, comportamento e cognição. Tendo tudo isso em vista, a teoria da autodeterminação nos traz a uma ideia de educação mais libertadora, que busca conhecer o aluno, assim como o que ele sente dentro do âmbito escolar e fora dele, motivando-o e mantendo relações de confiança e afeto que geram o interesse do aluno.

Deste modo, pudemos concluir, também, pela importância de uma pedagogia crítica que esteja de acordo com os direitos humanos e que se faz necessário adotar teorias pedagógicas que ofereçam apoio à ação educativa em Direitos Humanos. Apostamos na ideia de que a adoção de conceitos críticos como conscientização, autonomia, diálogo e emancipação, contribui para propiciar novas formas de investigação e de análise para o desenvolvimento do aluno. Podemos, assim, considerar que a prática pedagógica crítica em Direitos Humanos, ou seja, aquela que, entre outros assuntos, incorpore conceitos como autonomia e gestão à formação inicial, contribui também para um olhar diferenciado, que inclui o excluído, desconstruindo relações e 
práticas sociais históricas injustas, inclusive aquelas relativas à própria imagem do profissional licenciado em educação física.

Concluímos, desta maneira, que os artigos encontrados se assemelham no que tange à crítica ao sistema educacional, distribuindo-se em subtemas como: questões de gênero e sexualidade; aprendizagem científica e processos de socialização no ambiente escolar; educação pública laica; valorização da diversidade; qualidade de vida; inovação curricular; interculturalidade; reformas universitárias; responsabilidade social; estratégias de inclusão; políticas de equidade de gênero; qualidade das aprendizagens escolares; papel do professor como um adulto de referência; especificidades da educação pública; motivação tanto do professor como do aluno; saúde, dentre outros.

A partir dos dados encontrados pudemos concluir que quando, na busca, associase as quatro palavras chaves autonomia - currículo - gestão - educação física, são poucas as pesquisas encontradas que realmente trazem as quatro como fundamentais. No momento em que diminuímos a quantidade de palavras chaves, os resultados aumentam significativamente. Para nós, descobrir, por meio de uma pesquisa bibliográfica na área de graduação em educação física, que a mesma não é associada a autonomia, currículo e gestão, pelo menos não explicitamente, nos revela que a área não atrela tais palavras como fundamentais para o desenvolvimento do campo, assim como para seus futuros profissionais.

Tendo em vista tudo que foi dito anteriormente, é importante existir um currículo que possibilite liberdade e gerência ao professor e aluno, assim como que instigue à autonomia.

Dada a baixa incidência de produção encontrada nas duas plataformas analisadas, fomos ao encontro do currículo da Escola de Educação Física e Desportos (EEFD) da Universidade Federal do Rio de Janeiro, curso de Licenciatura, disponível no site $^{7}$ da própria faculdade. O currículo analisado é o currículo atual da EEFD, que não sofre nenhuma alteração desde 2007 e possui 38 disciplinas. Infelizmente, não foi possível encontrar nenhuma das três palavras chave: "autonomia", "gestão" e "currículo" nas ementas das 38 disciplinas, o que nos revela um dado um tanto quanto preocupante.

O projeto pedagógico, feito em 2006 e utilizado até hoje, tem como objetivos estabelecidos para o curso:

7 Disponível em: https://www.eefd.ufrj.br/files/Projeto_Pedag\%C3\%B3gico\%20200pdf. Acesso em: 11 de jun 2019. 
Gerais: Preparar professores licenciados em Educação Física para uma atuação reflexiva, crítica, transformadora e democrática em função dos direitos, necessidades e interesses da maioria da população brasileira; - Estimular o processo de formação contínua dos futuros professores, através do interesse pela busca e produção de novos conhecimentos advindos de pesquisas científicas e práticas de extensão, visando seu aperfeiçoamento permanente.

Específicos: Desenvolver as competências técnicas para o planejamento, execução e avaliação das atividades docentes na área de Educação Física, dirigidas à Educação Infantil, ao Ensino Fundamental e ao Ensino Médio (UFRJ, 2006).

Como se vê, não foi possível encontrar menção às palavras-chave. Além disso, observamos que o objetivo específico do curso pretende desenvolver competências técnicas, deixando, aparentemente, de lado, a necessidade de se formar um professor que saiba da importância do seu papel dentro das escolas para com seus alunos. Em nossa percepção, o caráter técnico não deve falar mais alto nem ficar abaixo de outras habilidades, pois é justamente neste momento que a nota que a criança tira na prova pode passar a ser vista como tendo mais valor que o aprendizado como um todo. Assim, defendemos que todas as habilidades deveriam se equiparar.

\section{Considerações finais}

A importância do professor de Educação Física em entender que o papel dele vai muito além de "rolar a bola" é o cerne de nossa preocupação. As qualificações desse professor, assim como sua constante atualização, fazem com que o mesmo esteja embasado e saiba utilizar todo o arsenal construído na sua contínua formação. Jogar a bola e deixar o jogo correr sem se preocupar com as particularidades e individualidades que envolvem uma turma é uma atitude simplista demais. Tal atitude deixa a desejar no que tange a uma educação diferenciada e que preze pelo amplo desenvolvimento técnico, cognitivo e motor, sem dúvida; mas que não deixe de fora o lado cultural, afetivo, político e autônomo de cada aluno.

Por meio dos dados obtivemos respostas fundamentais, mesmo que as respostas tenham vindo da baixa incidência dos resultados. Constatamos que poucos artigos possuem as palavras-chave de nosso interesse, a saber: autonomia, gestão e currículo. Para nós, esse resultado revela o quanto o tema em questão é pouco explorado, uma vez que a quantidade de produções nesta área é rara. 
Sendo assim, concluímos que é fundamental que a educação seja um lugar plural (e, junto com ela, a formação inicial de professores de Educação Física), que saiba a importância de estimular o futuro professor com um pensamento autônomo, em que o professor, assim como o aluno, tenham sua parte na gerência da disciplina e em que o currículo seja sempre adaptado e atualizado para que ele preveja o que há de melhor para o sistema educacional.

AGRADECIMENTOS: CNPq, CAPES e FAPERJ

\section{REFERÊNCIAS}

BOZYIGIT, E. Sports Management Education in Turkish Universities, Analysis of Undergraduate Programs. Journal of Sports Science 5, 2017, p. 345-348. DOI: 10.17265/2332-7839/2017.06.007

DIAS, E. de P. Conceitos de Gestão a Administração: uma revisão crítica. Revista Eletrônica de Administração, v. 1, 1. ed., jul./dez. 2002. Disponível em: http://legacy.unifacef.com.br/rea/edicao01/ed01_art01.pdf. Acesso em: 26 ago. 2012.

BETTIA, M.; KNIJNIKB, J.; VENÂNCIO, L.; NETO, L. S. In: search of the autonomous and critical individual: a philosophical and pedagogical analysis of the physical education curriculum of São Paulo (Brazil). Physical Education and Sport Pedagogy, 2015, v. 20, n. 4, p. $427-441$.

LARA, M.; STAREPRAVO, F. A.; MIRANDA, A. C. M.; SOUZA, F. M. QUALIDADE NA EDUCAÇÃO/EDUCAÇÃO FÍSICA ESCOLAR LATINOAMERICANA: ENCONTRO DE VOZES NADA DISSONANTES. J. Phys. Educ., v. 29, e2929, 2018.

MITCHELL, F.; GRAY, S.; INCHLEY, J. 'This choice thing really works'. Changes in experiences and engagement of adolescent girls in physical education classes, during a school-based physical activity programme. Physical Education and Sport Pedagogy, 2015 , v. 20 , n. 6 , p. $593-611$.

NEVES, R. (1997). Educação Física no 1.o Ciclo Ensino Básico - do Baldio Pedagógico À Construção Curricular. Actas Das III Jornadas Luso-Hispânicas de Educação Física no 1.o Ciclo do Ensino Básico, Seia. DRE - Centro e CAE - Guarda.

NEVES, R. (1999). "Educação Física no 1.o Ciclo do Ensino Básico - das necessidades de formação à profissionalidade docente". Actas das $\mathbf{V}$ Jornadas Luso-Hispânicas de Educação Física no 1.o CEB (CD-R), Celorico da Beira, DRE - CENTRO e CAE Guarda.

FREIRE, P. Pedagogia da Autonomia. São Paulo: Paz e Terra, 1996 
HARARI, Y. N. Sapiens - uma breve história da humanidade. Porto Alegre: LP\&M, 2017.

KILBORN, M.; LORUSSO, J.; FRANCIS, N. An analysis of Canadian physical education curricula. European Physical Education Review 2016, v. 22, n. 1, p. 23-46, a The Author(s) 2015

KIRSTEN, P. Architectures of practice: constraining or enabling PE in primary schools, Education 3-13, 44:5, 537-546. DOI: 10.1080/03004279.2016.1169484, 2016.

MENEGHETTI, F. K. O que é um ensaio-teórico?. Revista de Administração Contemporânea, v. 15, n. 2, p. 320-332, 2011.

NOGUEIRA, J. A. D.; BOSI, M. L. M. (2016). Saúde Coletiva e Educação Física: distanciamentos e interfaces. Ciência e Saúde Coletiva, v. 22, n. 6, p. 1913-1922, 2017.

PARO, V. H. Diretor Escolar: educador ou gerente? São Paulo: Cortez, 2015.

PARO, V. H. Administração Escolar: introdução crítica. 17. ed. São Paulo: Cortez, 2012.

RÉVILLION, A. S. P. A utilização de pesquisas exploratórias na área de marketing. Revista Interdisciplinar de Marketing, v. 2, n. 2, p. 21-37, 2015.

RYAN, R. M.; DECI, E. L. (2000b). Self-determination theory and the facilitation of intrinsic motivation, social development, and well-being. American Psychologist, v. 55 , n. 1, p. $68-78$.

SANTOS, M. P. dos. Dialogando sobre inclusão em educação: contando casos (e descasos). Curitiba: CRV, 2013.

SAVIANI, D. Educação escolar, currículo e sociedade: o problema da base nacional comum curricular. movimento-revista de educação, n. 4, 2016.

SENNA, M. (Re)visitando culturas, políticas e práticas de inclusão em educação no nível da gestão municipal de educação: a $2^{\text {a }}$ CRE em ação. Rio de Janeiro, 2017. Dissertação (Mestrado em Educação). Faculdade de Educação, Universidade Federal do Rio de Janeiro, Rio de Janeiro, 2017.

SVENDSEN, A. M.; SVENDSEN, J. T. (2016) Teacher or coach? How logics from the field of sports contribute to the construction of knowledge in physical education teacher education pedagogical discourse through educational texts. Sport, Education and Society, v. 21, n. 5, p. 796-810, DOI: 10.1080/13573322.2014.956713

UFRJ, 2006. Projeto Pedagógico Licenciatura em Educação Física (Reformulação Curricular). Disponível em:

https://www.eefd.ufrj.br/files/Projeto_Pedag\%C3\%B3gico\%202006.pdf. Acesso em: 11 jun. 2019. 


\section{Como referenciar este artigo}

SILVA, Allana Glauco; SANTOS, Mônica Pereira dos; SENNA, Manoella. Gestão e autonomia no currículo de Educação Física: Caminhos para a desconstrução da imagem de "jogadores de bola". Revista on line de Política e Gestão Educacional, Araraquara, v. 23, n. esp. 1, p. 936-951, out. 2019. E-ISSN:1519-9029.DOI: https://doi.org/10.22633/rpge.v23iesp.1.13021

Submetido em: 10/05/2019

Revisões requeridas: $14 / 06 / 2019$

Aprovado em: 10/08/2019

Publicado em: 01/10/2019 